\title{
RIQUEZA EM ÓLEO NAS SEMENTES, AMÊN- DOAS E CASCAS DAS BAGAS DE MAMONA
}

\author{
J. T. A. GURGEL e O. VALSECHI \\ Docentes-Livres e Assistentes da E. S. A. "Luiz de Queiroz" \\ Universidade de S. Paulo
}

\begin{abstract}
INDICE

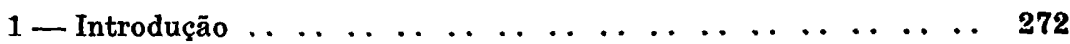

2 - Material e Método . . . . . . . . . . . . . . . . . . . 274

3 - Variabilidade do conteúdo em óleo na semente, na amêndoa e $n a$ casca $\ldots \ldots \ldots \ldots \ldots$

4 - Decomposição do êrro padrão total . $\ldots \ldots \ldots \ldots$

5 - Decomposição com agrupamentos . . . . . . . . . . . . . . 279

6 - Análise da correlação . . . . . . . . . . . . . . . . . . . . 280

7 - Resumo e Conclusões . . . . . . . . . . . . . . . . . . . . . 281

8 - Summary and Conclusions .. $\ldots \ldots \ldots \ldots$

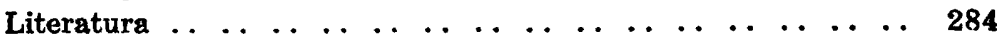

Os autores agradecem aos professores F. G. Brieger e Jayme Rocha de Almeida as sugestões apresentadas no decorrer do trabalho e a leitura do manuscrito. 


\section{1 - INTRODUÇAOO}

Refletindo-se, hoje, sôbre as múltiplas utilidades do óleo de rícino (mamona) fàcilmente chega-se a conclusão que o mesmo pode e deve estar colocado entre os principais do mundr. Realmente, para que se possa ter uma leve idéia de sua importância basta que se alinhem aquí, sucintamente, algumas de suas aplicações, como o faremos, seguindo de perto JAMIESON (4) no seu livro "Vegetable fats and oils".

O óleo de primeira (extraído por expressão a frio e refinado) \& usado para fins técnicos, medicinais e, com alguma extensão, como lubrificante especial; enquanto que, o de terceira (o comércio, desde há muito tempo, não reconhece qualquer óleo de rícino, como de segunda) é usado sòmente para fins técnicos, tanto no estado crú, como grosseiramente refinado.

Uma grande parte é convertida em óleo de rícino sulfonadi, conhecido sob a denominação de "óleo vermelho perú" (turkey red oil) devido ao seu emprêgo nas fábricas de tecidos de algodão, juntamente com a alizarina, como corante. Tais óleos säo usados para dar côres mais claras e brilhantes aos tecidos de algodão, linho e sêda e, ainda, na indústria de couros.

O óleo, "in natura" é empregado na manufatura de alguns sabōes especiais, como por exemplo, transparentes, e para indústrias téxteis. Segundo SPASSKII, citado por JAMIESON (4), os sabôes, nestas condições, espumam mais e são mais solúveis em água fria.

E' empregado, também, "in natura", na indústria de couros sintéticos, papéis de sêda espêssos e tintas para tipografias.

Foi usado, até há pouco como lubrificante para motores de avião, de alta rotação, com resfriamento de ar, sendo até hoje, em alguns paises de clima tropical, usado como lubrificante de maquinária pesada, inclusive locomotivas.

Considerável quantidade de óleo encontra aplicação na manufatura de nitro-celulóse, sendo também empregado como matéria prima no preparo de essências aromáticas, juntamente com o ácido sebácico.

Outra grande quantidade é convertida em óleo de rícino desidratado, o qual, sob várias denominações (Castung, Collanoll, Isoline, Oleo P. G. D., Synthenol, Synouryn, etc.), no comércio. é vendido como um óleo sicativo. Esta aplicação tornou-se possível em consequência do conhecimento que se tinha de que o ácido ricinolêico - principal constituinte do óleo de rícin: - podia ser desidratado pelo aquecimento, quando em presença de um catalisador apropriado. O grupo hidróxilo do ácido, sob estas condições, combina-se com um átomo de hidro- 
gênio de um grupo adjacente para dar formação a uma molécula de água, produzindo uma dúplice liga. Mais recentemente, verificou-se que o processo podia ser simplificado, usando-se diretamente o óleo, com produção de ácidos linolêicos 9,12 e. 9,11 na razão de três para um. A quantidade de ácido conjugado octodecadienóico 9,11 dos produtos comerciais varia de 17 a $26 \%$, enquanto que o isômero 9,12 de 59 a $64 \%$. Tais produtos acusam um índice de iodo (Wijs) que varia de 109 a 140; os índices de dieno - medida da quantidade de ácido linolêico, com dúplices ligas conjugadas e, ao qual se atribui considerável importância em relação à sicatividade do óleo - varia entre 13 a 22. O óleo desidratado, tal como é, ou após sofrer o devido encorporamento (óleos soprados e repousados) tem encontrado larga aplicação no preparo de esmaltes, tintas e vernizes, incluindo os vernizes próprios para estamparia e litografia, substituindo, portanto, em parte, o óleo de tungue. Aliás, o óleo de rícinci desidratado, tem preferência ao óleo de tungue, nos casos onde à retenção da côr e a flexibilidade de camadas finas sejam de importância.

Pelo fato de não amarelecer com o tempo, MUNDY, citad) por JAMIESON (4), relata a sua grande utilidade na fabricação de certas matérias plásticas, como substituto do vidro de janela

O óleo de rícino soprado é utilizado, como auxiliar, no preparc: de pastas e de lacas coloridas e para outros fins onde um óleo plastificante se faça necessário.

Durante o ano de 1937 o "Munzel Chemical Works" de Hausen, na Suissa (4) garantiu uma patente sôbre o processo para a produção de "TRIENOL" (óleo sintético de tungue) do óleo de rícino. A primeira fase do processo compreende uma desidratação que produz dúplices ligas conjugadas no ácido ricinolêico. Depois da remoção do ácido di-hidroxiestearico, do olêico, etc. - constituintes normais do óleo de rícino - o produto, chamado "DIENOL" é tratado com ácido hipocloroso, para a produção de dúplices e tríplices ligas conjugadas. O clóro introduzido e o grupo hidróxilo são eliminados. O Trienol, apresenta as seguintes principais propriedades: pêso específico a $20^{\circ} \mathrm{C} ., 0,928 ; \mathrm{N}_{\mathrm{D}}^{20}, 1,5175$; índice de iodo, 244,5; índice de saponificação, 190; índice de acidês, 8,2; matéria insaponificável, $0,57 \%$ e teste de calor, 12 minutos.

Pelo pouco que foi exposto já se pode avaliar da importância técnica e econômica do óleo de rícino. Por essa razão, as Secções de Genética e de Química Tecnológica da Escola Superior de Agricultura "Luiz de Queiroz", da Universidade de S. Paulo, resolveram, em cooperação, efetuar um estudo relativo 
à determinação do óleo nas sementes, nas amêndoas e nas cascas das bagas de mamona, visando com isso obter um critério para a seleção de variedades.

\section{2 - MATERIAL E MÉTODO}

As sementes de mamoneira usadas para êste estudo foram colhidas da coleção de progênies e variedades da Secção de Genética da Escola Superior de Agricultura "Luiz de Queiroz" e provêm de linhas pràticamente puras, com 4 a 5 autofecundações. Os frutos eram secos em um secador de ventilação forçada, mantendo-se sempre o mesmo critério para tôdas as amostras; o critério da perda de umidade foi mantido pelo contrôle por termohigrógrafo Fuess. Após isto, os frutos das variedades indeiscentes eram quebrados a mão e depois das sementes limpas, procederam-se as determinações do pêso, das medidas das sementes e da riqueza em óleo da semente, da amêndoa e da casca. A determinação do conteúdo em óleo das cascas, à primeira vista extranho, justifica-se pelo fato de que, em algumas variedades, a amêndoa não se destaca bem na região da carúncu la: para certos fins industriais, o descorticamento das sementes é praticado, sendo de interêsse para os nossos trabalhos de melhoramento, saber quais as variedades que perdem menos óleo $\mathrm{n}:$ seu processamento.

\section{1-Determinação das dimensões e do pêso das sementes}

As medidas das sementes foram tomadas em milímetros, utilizando-se um aparelho simples de madeira, à semelhança de um paquímetro; os resultados dessas determinações encontramse no quadro 1 .

Como critério de classificação, as bagas de mamona foram divididas de acôrdo com o pêso de 100 sementes, em pequenas, médias $e$ grandes; sementes pequenas são aquelas que têm um pês's no máximo até $30 \mathrm{~g}$ por 100 sementes; médias, as que têm um pêsc de 100 sementes variando entre 30 a $60 \mathrm{~g}$ e grandes, as que têm um pêso superior a $60 \mathrm{~g}$ por 100 sementes (quadro 1).

De todos os critérios que tentamos para classificar as semertes da mamoneira, o pêso de 100 sementes foi o que achamos mais satisfatório, porque nunca encontramos tipos transgressivos, conforme se depreende do quadro 1; nesse mesmo quadrı, podemos notar que as dimensões das sementes não se prestam rnuito bem para a sua separação em classes, principalmente entre as médias e grandes. 
2.2.-Determinação da percentagem de óleo nas sementes

Para a determinação da percentagem de óleo usamos o metodo clássico, pelo extrator de Soxhlet, tendo como solvente o alcool etílico anidro. Procedemos da seguinte maneira : da amostra média retirávamos ao acaso 100 sementes. Dessas 100 sementes tomávamos um número suficiente para perfazer um total aproximadamente de 3 a $4 \mathrm{~g}$, que era pesado exatamente e, em seguida era triturado com areia grossa, até a obtenção de uma farinha solta. Esta era passada, agora quantitativamente, a um cartucho extrator, com o auxílio de um pincel, algodão e álcool. Procedia-se à extração durante 6 horas. A seguir, o óleo era tornado isento de álcool por evaporação em banho-Maria e secagem em estufa a $100^{\circ} \mathrm{C}$., durante 2 horas; procedia-se à sua pesagen, depois de resfriado em dissecador apropriado.

\section{3 - Determinação da percentagem de óleo nas amêndoas}

Das restantes sementes procedia-se ao descorticamento, separando-se de um lado as amêndoas e de outro, as cascas. Umas tantas amêndoas, retiradas ao acaso, eram pesadas exatamente entre 3 a $4 \mathrm{~g}$, procedendo-se com as mesmas, da mesma forma que para o caso anterior.

\section{4 - Determinação da percentagem de óleo nas cascas}

Das cascas separadas, da operação anterior, retirávamos, também: ao acaso, um pêso certo entre 3 a $4 \mathrm{~g}$. Em seguida, as mesmas eram trituradas num almofariz, em ausência de areia, e passadas ao cartucho extrator. As operações que se seguiam foram idênticas as do primeiro processo.

\section{3-VARIABILIDADE DO CONTEÚDO EM OLEO NA SEMENTE, NA AMENDOA E NA CASCA}

Os dados do conteúdo em óleo das sementes, da amêndoa e da casca, expressos em percentagens, constam do quadro 1, que foi organizado de acôrdo com o critério, de separar as mamonas em 3 classes, isto é, pequenas, médias e grandes; dentro de cada classe as variedades foram colocadas pela ordem crescente de porcentagem de óleo da semente. Podemos notar que existe umà certa variabilidade na porcentagem de óleo nas partes analisadas de semente das diferentes variedades; assim, na 3a. coluna relativa a porcentagem de óleo nas sementes, o menor va- 
lor, de $45,46 \%$ é da variedade $n .1452$ de sementes pequenas e o maior valor, de $57,27 \%$ é da variedade n. 1013 de sementes médias, sendo a amplitude de variação de $11,81 \%$. Diferenças nẫo muito apreciáveis encontramos dentro das variedades, de um mesmo grupo, no que diz respeito à percentagem de óleo na semente, sendo de $6,52 \%$ nas variedades de sementes pequenas, de $10,84 \%$ nas variedades de sementes médias e de $6,71 \%$ nas variedades de sementes grandes. Quanto à porcentagem de óleo da amêndoa encontramos também uma variabilidade acentuada, com uma diferença de $15,38 \%$ dos tipos extremos, sendo a variedade mais pobre a de $n$. 1452 de sementes pequenas e a mais rica a de n. 1013 de sementes médias; aliás, as mesmas variedades respectivamente mais pobres e mais ricas no conteúdo em óleo da semente. Também aquí há bastante variação dentro de cada tipo de semente, pequenas, médias e grandes, send'; a diferença entre os tipos extremos, respectivamente de $9,85 \%, 12,65 \%$ e $8,17 \%$.

Com relação a porcentagem de óleo na casca vemos que elä é bastante variável e que não tem relação com o tipo da semer:te, podendo-se encontrar valores altos e baixos dentro de cada tipo; a diferença entre os tipos extremos foi de 4,79\%.

Se analisarmos individualmente as porcentagens de óleo obtido para as 28 variedades, iremos encontrar valores bem altos, chegando até $57,27 \%$; contudo, a fim de comparar os resultados por nós obtidos com outros autores, devemos tomar os valores médios para cada tipo, e não os extremos (quadro 1).

Entre nós PAULA (6), determinando a riqueza em óleo nas sementes de 20 variedades de mamoneira, na antiga Estação Experimental de Belo Horizonte, encontrou valores extremos de 41,18 e 52,55\%; MENDES (5), do Instituto Agronômico d $\epsilon$ Campinas, trabalhando com 45 variedades, achou para valores mínimo e máximo, respectivamente 36,34 e $51,92 \%$. BRIEGER e GURGEL (2) acharam para valores extremos 32,21 e $52,83 \%$.

Nas determinações feitas no estrangeiro, iremos citar apenas as publicações de ZAPPI-RECORDATI (7) e do Imperial Institute (1) de Londres:

Dados obtidos por ZAPPI-RECORDATI :

fndia

Africa do Norte

Africa Ocidental

Madagascar
$42,2-52,3 \%$

$46,2-51,3 \%$

$41,4-49,2 \%$

$40,5-47,1 \%$ 
Dados do Imperial Institute, Londres:

\section{Uganda}

Sudão

Rhodesia

Fiji

$$
\begin{aligned}
& 48,0-50,8 \% \\
& 41,0=48,7 \% \\
& 39,6-52,2 \% \\
& 47,4-49,6 \%
\end{aligned}
$$

A comparação dos valores médios por nós obtidos para a percentagem em óleo na semente está em concordância com os autores acima citados; contudo, temos em nossa coleção variedades com teores bem mais altos daqueles acima citados, extraidos da literatura.

\section{4 - DECOMPOSIÇÃO DO ERRO PADRÃO TOTAL}

A fim de termos uma idéia da variabilidade das determinações de óleo na semente, na amêndoa e na casca, calculamos os coeficientes de variação, cujos resultados são dados no quadro 2 Podemos notar que para a percentagem de óleo na semente e na amêndoa e para os três tipos de sementes, o coeficiente de variação está pràticamente entre 5 e $7 \%$; êstes valores sãr bem baixos, o que nos indica que as linhagens de mamoneira estudadas são pouco variáveis, quanto a percentagem de ólen na semente e na amêndoa.

Todavia, para a percentagem de óleo na casca o coeficiente de variação é muito alto, entre 50 a $60 \%$, tanto para as sementes pequenas, médias e grandes; portanto, concluimos que em relação a percentagem de óleo que fica aderente à casca, as linhagens estudadas diferem muito entre si.

Fazendo-se a decomposição do êrro total da percentagem de óleo para a semente, amêndoa e casca em êrro entre tipos e e êrro residual, notamos no quadro 3, que apenas para a percentagem de óleo na semente é que houve significância entre os três tipos considerados, no nível de $5 \%$ de probabilidade; por outras palavras, os grupos têm riquezas diferentes em relação a percentagem de óleo na semente.

Para a percentagem de óleo na semente, desde que há direfença entre os grupos, a fim de sabermos qual o mais rico, fizemos um teste de $t$; para o êrro da diferença entre média podemos aplicar a fórmula proposta por FISHER (3), usando um êrro médio balançado, que neste caso é o êrro residual.

$O$ teste de $t$ entre a percentagem de óleo das sementes pequenas e médias foi de $-2,10$ que com 18 graus de liberdade está justamente no nível de $5 \%$; entre a percentagem de óleo das sementes médias e grandes foi de $-2,02$ que com 17 graus 
de liberdade é insignificante, porém não muito longe do níve] de 5\%; finalmente, o valor de $t$ entre as percentagens de óleo das sementes pequenas e grandes foi de $-3,82$ que com 15 graus de liberdade é significante ao nível de $1 \%$. Portanto, em relação a percentagem de óleo na semente, o grupo de sementes grandes é mais rico que o de sementes médias e bem mais rico que o de sementes pequenas.

Esta última situação é de grande importância no melhoramento, pois sabendo que pràticamente a riqueza em óleo auaumenta com o tamanho da semente, devemos voltar as nossas vistas para o grupo das sementes médias e grandes, em vez de tentar as sementes pequenas; aliás, esta nossa conclusão está em desacôrdo com a opinião divulgada na lavoura, que diz justamente que as sementes menores é que são mais ricas em óleo A idêntica conclusão chegou PAULA (6) trabalhandc com variedades mineiras de mamoneiras.

Desde que provamos que os grupos são diferentes, para $a$ seleçãc das variedades torna-se necessário saber quais as mals ricas dentro dêles. O mais fácil é calcularmos os limites fiduciais para cada grupo, expostos no quadro 4; convém notar que para maior coordenação do cálculo, utilizamos-nos unicamente $d n$ êrro residual.

Analisando o quadro 4, notamos que apenas para o grupo de sementes médias encontramos a variedade $n$. 10132 - que tem uma porcentagem de óleo na semente maior do que o limite fiducial ao nivel de $5 \%$; isto nos indica que esta variedade pode ser considerada a melhor em relação a êste caracter. Nos demais grupos não podemos separar com segurança variedades mais ricas ou mais pobres do que a média.

Para calcularmos os limites fiduciais para as percentagens de óleo na amêndoa e na casca, desde que a análise de variação (quadrc 3) mostrou que não há diferença entre os grupos, o melhor é tomarmos a média geral e o êrro padrão total, pois êle é o que tem maior grau de liberdade. Assim, para a percentagem de óleo na amêndoa, com média geral de $66,11 \%$ e êrro padrão total de $\pm 3,41 \%$, os limites fiduciais são de 59,12 a $73,10 \%$ e 56,67 a $75,55 \%$ respectivamente para os níveis de $5 \%$ e $1 \%$ de probabilidade; para a percentagem de óleo na casca com média geral de $1,87 \%$ e êrro padrão total de $1,14 \%$, os limites riduciais são de 0 a $4,21 \%$ e 0 a $5,03 \%$, respectivamente para os níveis de $5 \%$ e $1 \%$. Devemos notar que para êste último cası a aplicação do teste é um pouco duvidosa, pois o limite fiducial mínimo dá resultados negativos, o que seria pràticamente impossível. 


\section{5 - DECOMPOSIÇAO COM AGRUPAMENTOS}

Como sabemos, na estatística quando temos uma série de valores, pode acontecer que a diferença entre valores consecutivos seja pequena: dizemos que êles formam uma distribuição contínua; acontece às vêzes que entre êles existem diferenças acentuadas, maximé quanto aos valores extremos; dizemos então que a distribuição é descontínua. Para a análise dos dois tipos, o tratamento estatístico a ser dispensado é diferente, pois enquanto que no tipo contínuo não temos justificação para agrupar os valores, no tipo descontínuo temos razões para isso, pris talvez tenhamos mais de uma distribuição superposta; todavia, sòmente uma decomposição com agrupamentos poderá resolver satisfatoriamente a questão.

Olhando-se para o quadro 1 , notamos que tanto para a percentagem de óleo na semente, como na amêndoa, os valores obtidos para as 28 variedades formam uma série contínua, náo havendo portanto justificativa para fazer agrupamentos. Entretanto, para a percentagem de óleo na casca, temos uma serie descontinua de valores na qual podemos notar no mínimo 5 grupos : o 1.0 de 0,43 a $1,34 \%$; o 2.0 de 1,62 a $1,84 \%$; o 3.0 de 2,13 a $2,68 \%$; o 4.0 de 3,44 a $3,62 \%$ e o 5.0 com um valor único de $5,22 \%$.

Para provar que temos 5 grupos diferentes, vamos fazer um novo teste "entre-dentro", cujos resultados são dados no quadri: 5; assim, temos o êrro entre os 5 grupos, o êrro dentro de cada grupo e um novo êrro residual. O êrro entre os 5 grupos foi calculado tomando-se a soma das correções de cada grupo (3a. coluna do quadro 5), e subtraindo-se a grande correção de 98,1377, dividindo-se por 4 e extraindo-se a raiz quadrída; o valor obtido para êsse êrro foi de $2,89 \%$.

Efetuando o teste de teta do êrro entre os 5 grupos, que e de $2,89 \%$ com êrro residual, de $0,25 \%$ obtivemos um valor muito alto, igual a 11,56, altamente significante. Isto prova que os 5 grupos são bem diferentes entre si. Doutro lado, os testes de teta dos êrros dentro dos grupos, quando comparados com o êrr ) residual, são todos insignificantes (quadro 5), mostrando-nos que os grupos são homogêneos.

A fim de corroborar a nossa hipótese de que temos 5 grupos diferentes quanto à percentagem de óleo na casca, podemos fazer ainda um teste de $t$ para as diferenças das médias de grupos consecutivos: os resultados dessas comparações são dados nc: quadro 6, no qual se nota que as médias dos grupos consecutivos são estatisticamente diferentes entre si. Portanto podemos dizer que a nossa série de 28 determinações da percenta- 
gem de óleo na casca não forma uma série contínua, mas ao contrário, forma uma série descontínua, com, no mínimo, 5 grupos äiferentes.

Assim, as variedades pertencentes ao grupo 4 e 5 , de ns. 1023-5, 1013-2, 1009-1 e 1076-4 deveriam ser excluidas do melhoramento, se porventura desejassemos variedades que não perdessem muito óleo no descorticamento, se bem que uma delas, a de n. 1013, seja a nossa variedade mais rica em óleo na semente e na amêndoa.

\section{6 - ANALISE DA CORRELAÇÃO}

Desde que temos três diferentes determinações da percentagen de óleo na semente, na amêndoa e na casca das 28 variedades estudadas, seria interessante sabermos se por ventura êsse valores são correlacionados entre si, pois isto teria valor prático na seleção das variedades.

Para 1sto, fizemos a correlação entre percentagem de óleo na semente e na amêndoa, percentagem de óleo na semente e na casca e finalmente percentagem de óleo na amêndoa e na casca Os resultados destas comparações são dados no quadro 7; podemos notar que apenas na comparação entre a percentagem de óleo na semente e na amêndoa encontramos alguns resultados significantes. Assim, vemos que no tipo de semente pequena, o valor do coeficiente de correlação de $+0,68$, é significante ao nível de $5 \%$ de probabilidade; podemos pois dizer que neste tipo de semente existe uma correlação positiva entre a percentagem de óleo da semente e da amêndoa. No tipo de semente média, encontramos uma correlação positiva de $+0,71$ significante no limite de $1 \%$ de probabilidade; portanto, aqui. podemos dizer, com mais segurança do que no caso anterior, que quanto mais rica em óleo for a semente, mais alto será o conteudo de óleo da amêndoa. Para o tipo de semente grande, o valor do coeficiente de correlação de $+0,55$ não foi significativo, talvez devidn an pequeno número de observações. Finalmente, para 0 total das 28 variedades o coeficiente de correlação de $+0,69$ foi significativo, no limite de $1 \%$ de probabilidade. Assim pois, de um modo geral, na mamona existe uma correlação positiva entr: a porcentagem de óleo na semente e na amêndoa, qualquer que seja o seu tamanho.

Os resultados da correlação entre a percentagem de óleo na semente e ná casca e a percentagem de óleo na amêndoa e na casca deram em todos os tipos de sementes, valores de correlação não significativos, com excepção apenas do total da 
percentàgem de óleo na amêndoa e na casca, que foi significante $n$ ) nivel de $5 \%$.

Êste resultado é bastante satisfatório para a seleção, umo vez que nos possibilita escolher as melhores sementes, quer seja'n pequenas, médias ou grandes, sem corrermos o risco de in. cidir justamente naquelas que deixem mais óleo na casca. Tal fato $\dot{\epsilon}$ importante, como já se frizou, quando por razões técnicas, nà extração do óleo houver necessidade de se efetuar o seu descorticamento.

\section{7 - RESUMO E CONCLUSÕES}

Os autores, justificando a razão do presente trabalho, citam as principais aplicações que o óleo de rícino tem na indústria, na medicina e na lubrificação. $\mathrm{Na}$ indústria é usado para da melhor côr aos tecidos (turkey red oil), é utilizado também na manufatura de sabões especiais, na fabricação de couros sintéticos, papel de sêda expesso e tintas; entra na composição da nitro-celulóse e como matéria prima no preparo de essências aromáticas. E' usado como óleo sicativo, em substituição ao tungue e faz parte ainda de inúmeros produtos sintéticos. $\mathrm{Na}$ mediciná é usado como purgativo. E' ainda utilizado como lubrificante nos motores de alta rotação, na maquinaria pesada, etc. En? vista das múltiplas aplicaçõ̃es que o óleo de mamona tem é que as Seç̧ões de Genética e Química Tecnológica desta Escola se congregaram para estudar a determinação do óleo nas sementes, nas amêndoas e nas cascas das bagas de mamona, visand. com isso obter um critério na seleção de variedades.

As sementes depois de colhidas, eram secas em um secado, de ventilação forçada, depois disso eram medidas com um paquínetro de madeira. Cada amostra consistia de 100 sementes colhidas ao acaso, das quais tomava-se um certo número até perfazer 3 a $4 \mathrm{~g}$. Nesta procedia-se a determinação do óleo. A extração do óleo era feita em extrator de Soxhlet, com álcooi. Fizeram-se as determinações separadamente nas sementes, nas amêndoas e nas cascas (quadro 1).

De acôrdo com o pêso de 100 sementes, (quadro 1) as bagas de mamona foram divididas em 3 classes: pequenas, cor 1 pêsc de 100 sementes menor de $30 \mathrm{~g}$; médias com pêso de 100 sernentes, compreendido entre 30 a $60 \mathrm{~g}$ e grandes com pêso de 100 sementes maior do que $60 \mathrm{~g}$; os resultados da análise da percentagem de óleo são dados no quadro 1. Para se ter uma idéia da variação da percentagem de óleo dentro do grupo das sementes pequenas, médias e grandes, foi determinado o coe-ficiente de variação para cada classe (quadro 2). A pərcenta- 
gem de óleo na semente e na amêndoa nas 3 classes é pouco va riável, com coeficiente de variação menor do que $10 \%$; a percentagem de óieo na casca foi excessivamente variável nas 3 classes, com coeficiente de variação maior do que $50 \%$. As com:parações feitas entre os resultados obtidos neste trabalho com os da literatura, mostram concordância quanto aos valores médios, havendo discordância entre os valores extremos.

A análise de variância feita para o êrro padrão total da percentagem de óleo da semente, da amêndoa e da casca para os 3 tipos de sementes, pequena, média e grande (quadro 3) mostroù que apenas para a percentagem de óleo da semente é que houve diferença entre os tipos de mamona estudados; 0 grupo de sementes grandes é pouco mais rico que o de sementes niédias e muito mais rico que o de sementes pequenas, enquanto que também as sementes médias são estatisticamenie mats ricás em óleo na semente do que as sementes pequenas. Foram calculados os limites fiduciais (quadro 4) para a percentagem de óleo na semente para cada classe, notando-se que uma variedade, de número 1013-2 tem uma riqueza em óleu significantemente maior do que a média do seu grupo, do nível de 5\%. Para a percentagem de óleo na amendoa todos os valores obtidos representam uma variação ao acaso da média geral.

Foi mostrado que a variação da percentagem de óleo na casca segue uma distribuição assimétrica e apresenta grande variabilidade; foi possível mostrar também que há cinco grupos es-. tatisticamente diferentes entre sí. De acôrdo com os resultados obtidos deve-se eliminar as variedades que percam muito óleo (mais do que 3\%) no descorticamento. Todavia, cumpre lembrar que nâo é muito comum na indústria extrair-se o óleo de sementes descorticadas, razão pela qual essa observação perde um poucc seu valor.

Existe uma correlação positiva significante entre a percentagem. de óleo na semente e na amêndoa para os tipos de sementes pequena e média, não se dando o mesmo para as sementes grandes; tomando-se em conjunto os 3 tipos de sementes, a correlação é significante no nível de $1 \%$. Não se encontraram correlações positivas entre percentagem de óleo na semente e na casca, e entre a amêndoa e casca; neste último caso, somente tomando-se os 3 tipos em conjunto é que foi notada uma correlação positiva significante no nível de $5 \%$. Aliás, êste último resultado confirma que algumas variedades mais ricas, quando descorticadas, têm uma tendência para perder mais óleo na casca. 
Finalmente, para fins de melhoramento, visando-se a riquequeza em óleo na semente, recomenda-se escolher variedades de sementes médias e grandes.

\section{SUMMARY AND CONCLUSIONS}

This paper is a joined publication of the Depts. of Genetics and of Technology, of the E. S. A. "Luiz de Queiroz", Universidade de São Paulo, and deals with the variation of the percentage oil content in the whole seeds, the embryos and the seed-coat of 28 varieties of castor-beans (Ricinus communis, L.).

Primarily, the authors, as a justification of this paper, make reference to the applications which castor-oil has in industry, medicine, etc.

In accordance with the weight of 100 seeds, the varieties: of castor-beans were classified into 3 classes : small seeds (100 seeds less than $30 \mathrm{~g}$ ), medium seeds (100 seeds between $30 \mathrm{~g}$ and 60 ) and large seeds (100 seeds more than $60 \mathrm{~g}$ ). The percentage of oil in the seed, embryo and seed-coat, the dimensions of the seeds and the weight of 100 seeds are given for every variety in table 1 .

In order to obtain an estimate of the variability for the methods of determination of the oil percentage, in the 3 differents parts of the seeds and also in the 3 groups of seeds, the coefficient of variability was calculate (table 2). It is showed that the variation in the seed and embryo is low and that in the seed-coat is very high.

The analysis of variance, with regard to the difference: aming the 3 types of seeds (small, medium and large), amons the 3 parts of the seed (whole seed, embryo and seed-coat) and residual error, is given in table 3. Only, the oil content of whole seeds among types of seeds was significant at the 5\% level. The $t$ test among the correspondent means is not significant for the difference between medium and large seeds is significant between both these types (medium and large) and snall seeds.

The fiducial limits in relation to the mean of the oil percentage in the 3 differents types of seed, show that there is one variety (n. 1013-2), which has a percentage of oil, in the mediun: type of seed, significantly at the $5 \%$ level (table 4), higher than the general mean.

Since the distribution of the percentage of oil in the seedcoat is discontinuous, 5 groups were established (table 5). Ail the differences between groups are significant (table 6). For 
practical purposes, when we have to remove the seed coat, one should eijminate those varieties which loose at least $3 \%$ of oil by this procedure.

There is a significant linear correlation at $5 \%$ level between the percentage of oil in the seed and in the embryo, of the small and medium type of seeds (table 7), and also, when taking the 3 types together (lower part of table 7), one finds that the same is true. Also, the correlation between the percentages of oil in the embryo and in the seed-coat of the 3 types together is significant at $5 \%$ level.

According to the results obtained in relation to the percentage in 28 varieties studied, it can be recommended, for breeding purposes, to work only with those varieties which belong to the medium and the large types of seeds.

\section{LITERATURA}

1-ANONIMO - Castor seed : its production and utilization. Bulletin of the Imperial Institute 28: 30-46, 1930.

2-BRIEGER, F. G. e J. T. A. GURGEL - Experiências preliminares sôbre a mamoneira (Ricinus communis, L.). Revista da Agricultura 15: 229-248, 1940.

3-FISHER - Em Statistical Methods for Research Workers. 10th ed. Oliver and Boyd, Edinburgh, 1946.

4-JAMIESON, G. S. - Em Vegetable Fats and Oils. 2and ed Reinhold Publishing Corp. New York, 1943.

5) -- MENDES, P. - A mamoneira - Generalidades e instruções para a sua cultura. Boletim n. 19, 28 pgs,. Instituto Agronômico do Estado, Campinas, 1938.

6-PAULA, E. VIANNA de - Como cultivar a mamona, 22 pgs, Secretaria da Agricultura de Minas Gerais, 1937.

7-ZAPPI-RECORDATI, A. - Il Ricino - Nome pratiche di coltivazione, 46 pgs. Ramo editoriale degli Agricoltori. T1pografia Federazioni Italiana, Roma, 1936. 
QUADRO 1

Resultado da análise da porcentagem de óleo, das dimensões e do pêso das sementes em 28 variedades $d:$ mamoneiras

\begin{tabular}{|c|c|c|c|c|c|c|c|c|c|c|}
\hline \multirow{2}{*}{$\begin{array}{l}\text { Tipo de } \\
\text { semente }\end{array}$} & \multirow{2}{*}{$\begin{array}{l}N^{\circ} \text {. da } \\
\text { família }\end{array}$} & \multicolumn{5}{|c|}{ Riqueza em óleo } & \multicolumn{3}{|c|}{ Dimensão das sementes } & \multirow{2}{*}{$\begin{array}{l}\text { Pêso de } 100 \\
\text { sementes }\end{array}$} \\
\hline & & na & semente & na & amêndoa & na casca & Compr. & Larg. & Espess. & \\
\hline Pequena & $\begin{array}{l}1452 \\
1073-1 \\
1002-2 \\
1003-1 \\
1005-6 \\
1048-1 \\
1079-1 \\
1009-1 \\
1008-1 \\
\end{array}$ & & $\begin{array}{c}\% \\
45,46 \\
45,62 \\
46,21 \\
46,41 \\
47,87 \\
49,09 \\
49,18 \\
50,96 \\
51,98 \\
\end{array}$ & & $\begin{array}{c}\% \\
57,57 \\
65,14 \\
63,95 \\
61,79 \\
67,35 \\
65,59 \\
64,67 \\
66,76 \\
67,42 \\
\end{array}$ & $\begin{array}{l}\% \\
2,38 \\
1,62 \\
0,78 \\
0,43 \\
2,13 \\
1,79 \\
0,58 \\
3,62 \\
2,40 \\
\end{array}$ & $\begin{array}{c}\mathrm{mm} \\
11,7 \\
12,2 \\
10,8 \\
10,0 \\
11,7 \\
11,8 \\
11,6 \\
10,9 \\
12,6 \\
\end{array}$ & $\begin{array}{c}\mathrm{mm} \\
7,1 \\
7,3 \\
6,9 \\
7,0 \\
7,0 \\
7,8 \\
7,2 \\
6,6 \\
7,0 \\
\end{array}$ & $\begin{array}{c}\mathrm{mm} \\
5,1 \\
5,5 \\
5,0 \\
5,0 \\
5,0 \\
5,6 \\
5,1 \\
4,9 \\
5,1 \\
\end{array}$ & $\begin{array}{c}\mathrm{g} \\
16,7 \\
21,5 \\
16,0 \\
12,8 \\
19,7 \\
25,2 \\
20,7 \\
18,2 \\
22,2\end{array}$ \\
\hline \multicolumn{2}{|c|}{$\bar{x}$} & & 48,09 & & $\angle 7^{6} \nabla^{\prime}$ & 1,75 & 11,5 & 7,1 & 5,1 & 19,2 \\
\hline Média & $\begin{array}{l}1102-6 \\
1050-5 \\
1056-3 \\
1034-5 \\
1075-1 \\
1007-5 \\
1069-3 \\
1037-3 \\
1023-5 \\
1013-2 \\
1076-4 \\
\end{array}$ & & $\begin{array}{l}46,43 \\
48,12 \\
48,30 \\
48,33 \\
48,57 \\
49,31 \\
51,25 \\
52,92 \\
53,82 \\
54,72 \\
57,27 \\
\end{array}$ & & $\begin{array}{l}60,30 \\
64,57 \\
66,66 \\
63,77 \\
64,00 \\
68,29 \\
69,00 \\
70,01 \\
69,37 \\
68,92 \\
72,95 \\
\end{array}$ & $\begin{array}{l}0,97 \\
2,41 \\
2,68 \\
1,34 \\
0,90 \\
1,84 \\
5,22 \\
0,57 \\
1,25 \\
3,44 \\
3,45 \\
\end{array}$ & $\begin{array}{l}14,3 \\
13,9 \\
15,4 \\
14,6 \\
12,5 \\
14,2 \\
14,2 \\
14,5 \\
15,8 \\
17,1 \\
15,6 \\
\end{array}$ & $\begin{array}{r}9,6 \\
8,5 \\
9,6 \\
9,1 \\
10,1 \\
8,9 \\
8,4 \\
8,4 \\
9,5 \\
10,0 \\
9,7 \\
\end{array}$ & $\begin{array}{l}7,0 \\
6,8 \\
7,0 \\
7,0 \\
6,3 \\
6,0 \\
5,9 \\
5,7 \\
7,3 \\
6,5 \\
7,4 \\
\end{array}$ & $\begin{array}{l}45,5 \\
35,6 \\
43,5 \\
35,8 \\
38,4 \\
38,7 \\
36,4 \\
34,2 \\
48,4 \\
51,2 \\
43,0\end{array}$ \\
\hline \multicolumn{2}{|c|}{$\bar{x}$} & & 50,82 & & 67,08 & 2,19 & 14,7 & 9,3 & 6,6 & 41,0 \\
\hline Grande & $\begin{array}{l}1462 \\
1025-1 \\
1014-6 \\
1018-6 \\
1032-1 \\
1019-3 \\
1010-3 \\
1017-6 \\
\end{array}$ & & $\begin{array}{l}50,02 \\
50,31 \\
52,37 \\
52,87 \\
53,90 \\
55,85 \\
56,01 \\
56,73 \\
\end{array}$ & & $\begin{array}{l}62,72 \\
66,94 \\
65,16 \\
66,90 \\
66,07 \\
63,14 \\
71,13 \\
70,89 \\
\end{array}$ & $\begin{array}{l}0,85 \\
2,67 \\
1,34 \\
0,72 \\
1,08 \\
2,51 \\
2,25 \\
1,20 \\
\end{array}$ & $\begin{array}{l}17,9 \\
21,4 \\
19,4 \\
19,5 \\
18,7 \\
19,7 \\
16,1 \\
19,2 \\
\end{array}$ & $\begin{array}{l}13,6 \\
10,7 \\
14,2 \\
13,9 \\
14,1 \\
14,1 \\
11,9 \\
13,3\end{array}$ & $\begin{array}{r}8,0 \\
7,0 \\
8,1 \\
8,2 \\
8,0 \\
8,0 \\
7,3 \\
14,1 \\
\end{array}$ & $\begin{array}{l}81,7 \\
66,0 \\
86,5 \\
94,3 \\
84,5 \\
77,2 \\
59,6 \\
93,1 \\
\end{array}$ \\
\hline & & & 53,51 & & 66,62 & 1,58 & 19,0 & 8,0 & 7,8 & 80,4 \\
\hline
\end{tabular}

QUADRO 2

Determinação da média, êrro padrão e coeficiente de variação, para a porcentagem de óleo na semente, na amêndoa e na casca nos 3 tipos de sementes peauenas, médias e grandes

\begin{tabular}{|c|c|c|c|c|c|c|c|c|c|}
\hline \multirow{2}{*}{$\begin{array}{l}\% \text { de } \\
\text { óleo na }\end{array}$} & \multicolumn{3}{|c|}{ Semente pequena } & \multicolumn{3}{|c|}{ Semente média } & \multicolumn{3}{|c|}{ Semente grande } \\
\hline & $\bar{x}$ & $\theta$ & $0 \%$ & $\bar{x}$ & $\sigma$ & $\sigma \pi r$ & $\bar{x}$ & $\sigma$ & $\sigma \%$ \\
\hline $\begin{array}{l}\text { Semente } \\
\text { Amêndoa } \\
\text { Casca }\end{array}$ & $\begin{array}{r}48,09 \\
64,47 \\
1,75 \\
\end{array}$ & $\begin{array}{l} \pm 2,37 \\
\pm 3,14 \\
\pm 1,03\end{array}$ & $\begin{array}{l} \pm \quad 4,9 \\
\pm \quad 4,9 \\
\pm 58.9\end{array}$ & $\begin{array}{r}50,82 \\
67,08 \\
2.19 \\
\end{array}$ & $\begin{array}{l} \pm 3,42 \\
\pm 3,60 \\
\pm 1.42\end{array}$ & $\begin{array}{l} \pm \quad 6,7 \\
\pm \quad 5,4 \\
\pm 64.8\end{array}$ & $\begin{array}{r}53,51 \\
66,62 \\
1,58 \\
\end{array}$ & $\begin{array}{l} \pm 2,57 \\
\pm 3,13 \\
\pm 0,78\end{array}$ & $\begin{array}{l} \pm 4,8 \\
\pm 4,7 \\
\pm 49,4\end{array}$ \\
\hline
\end{tabular}

QUADRO 3

Análise da variância para a porcentagem de óleo na semente, na amêndoa e na casca das bagas de mamon̋

\begin{tabular}{|c|c|c|c|c|c|c|c|c|c|}
\hline \multirow{2}{*}{$\begin{array}{l}\text { Fontes de } \\
\text { variaçãao }\end{array}$} & \multicolumn{3}{|c|}{$\%$ óleo na semente } & \multicolumn{3}{|c|}{ \% óleo na amêndoa } & \multicolumn{3}{|c|}{ \% óleo na casca } \\
\hline & $\sigma$ & $\mathrm{nf}$ & $\vartheta$ & $\sigma$ & nf & $\vartheta$ & $\sigma$ & $\mathrm{nf}$ & $\vartheta$ \\
\hline $\begin{array}{l}\text { Êrro total } \\
\text { Êrro entre }\end{array}$ & $\pm 3,51$ & 27 & 1,21 & $\pm 3,41$ & 27 & 1,02 & $\pm 1,14$ & 27 & 0,99 \\
\hline Êrro & $\pm 7,90$ & 2 & $2,73^{*}$ & $\pm 4,27$ & 2 & 1,28 & $\pm 0,98$ & 2 & 0,85 \\
\hline residual & $\pm 2,89$ & 25 & - & $\pm 3,3 ?$ & 25 & - & $\pm 1,15$ & 25 & - \\
\hline $\begin{array}{l}\text { Médias } \\
\text { Gerais }\end{array}$ & \multicolumn{3}{|c|}{$50,71 \pm$} & \multicolumn{3}{|c|}{$66,11 \%$} & \multicolumn{3}{|c|}{$1,87 \%$} \\
\hline $\begin{array}{l}\text { Coef. de } \\
\text { var. }\end{array}$ & \multicolumn{3}{|c|}{$6,9 \%$} & \multicolumn{3}{|c|}{$5,2 \%$} & \multicolumn{3}{|c|}{$61,0 \%$} \\
\hline
\end{tabular}


QUADRO 4

Limites fiduciais para a porcentagem de óleo na semente, para os três tipos de bagas pequenas, médias e grandes

\begin{tabular}{|c|c|c|c|c|c|c|c|c|}
\hline $\begin{array}{l}\text { Porcentagem de } \\
\text { óleo na semente }\end{array}$ & $\begin{array}{l}\text { Nível de } \\
\text { Prob. }\end{array}$ & $\mathbf{t}$ & ${ }_{\mathrm{R}}^{\sigma}$ & t. $\sigma$ & $\bar{x}$ & Limites & fiduciais & $\begin{array}{l}\text { Variáveis } \\
\text { extremas }\end{array}$ \\
\hline Pequena & $\begin{array}{l}5 \% \\
1 \% \\
\end{array}$ & $\begin{array}{l}2,31 \\
2,36\end{array}$ & 2,89 & $\begin{array}{l}6,68 \\
9,71\end{array}$ & 48,09 & $\begin{array}{l}41,41 \\
38 ; 38 \\
\end{array}$ & $\begin{array}{r}-54,77 \\
-\quad 57,80\end{array}$ & $45,46-51,98$ \\
\hline Médja & $\begin{array}{l}5 \% \\
1 \% \\
\end{array}$ & $\begin{array}{l}2,23 \\
3,17 \\
\end{array}$ & 2,89 & 6,44 & 50,82 & $\begin{array}{l}44,38 \\
41,66\end{array}$ & $\begin{array}{r}-\quad 57,26 \\
-\quad 59,98\end{array}$ & $46,43-57,27$ \\
\hline Grande & $\begin{array}{l}5 \% \\
1 \%\end{array}$ & $\begin{array}{l}2,37 \\
3,50\end{array}$ & 2,89 & $\begin{array}{r}6,85 \\
10,12\end{array}$ & 53,51 & $\begin{array}{l}46,66 \\
43,39\end{array}$ & $\begin{array}{r}-60,36 \\
-\quad 63,63\end{array}$ & $50,02-56,73$ \\
\hline
\end{tabular}

QUADRO 5

Marchá do cálculo para os êrros dentro dos grupos e residual nos 5 grupos da porcentagem de óleo na casca

\begin{tabular}{|c|c|c|c|c|c|c|}
\hline Grupos & $\Sigma \mathrm{x}^{2}$ & $\mathrm{C}^{\prime}$ & $\bar{x}$ & $\mathrm{nf}$ & $\vartheta$ & $o \mathrm{D}$ Grupo \\
\hline $\begin{array}{l}1^{\circ} . \text { grupo } \\
2^{\circ} . \text { grupo } \\
3^{\circ} . \text { grupo } \\
4^{\circ} . \text { grupo } \\
5^{\circ} \text {. grupo }\end{array}$ & $\begin{array}{r}12,2065 \\
9,2141 \\
47,4433 \\
36,8405 \\
27,2484\end{array}$ & $\begin{array}{r}11,0954 \\
9,1875 \\
47,1906 \\
36,8200 \\
27,2484\end{array}$ & $\begin{array}{l}0,93 \% \\
1,75 \% \\
2,43 \% \\
3,50 \% \\
5,22 \%\end{array}$ & $\begin{array}{r}12 \\
2 \\
7 \\
2 \\
-\end{array}$ & $\begin{array}{l}1,20 \\
0,48 \\
0,76 \\
0,40\end{array}$ & $\begin{array}{l} \pm 0,30 \% \\
\pm 0,12 \% \\
\pm 0,19 \% \\
\pm 0,10 \% \\
\end{array}$ \\
\hline$\Sigma$ & 132,9528 & 131,5419 & - & 23 & - & $\sigma \mathrm{R}= \pm 0.25 \%$ \\
\hline
\end{tabular}

QUADRO 6

Resultados do teste de $t$ para as diferenças das médias consecutivas dos 5 grupos para a porcentagem de óleo na casca

\begin{tabular}{l|r|r|l|r}
\multicolumn{5}{|c|}{ porcentagem de óleo na casca } \\
\hline $\begin{array}{c}\text { Diferenca entre } \\
\text { os arupos }\end{array}$ & $\overline{\mathbf{x} 1}-\overline{\mathrm{x} 2}$ & $\sigma$ dif. & Teste de t & nf \\
\hline & & & & \\
1 e 2 & $-0,62$ & $\pm 0,16$ & $-3,88^{* *}$ & 14 \\
2 e 3 & $-0,68$ & $\pm 0,17$ & $-4,00^{* *}$ & 9 \\
3 e 4 & $-1,07$ & $\pm 0,17$ & $-6,29^{* *}$ & 9 \\
4 e 5 & $-1,72$ & $\pm 0,35$ & $-4,91^{*}$ & 2 \\
\hline
\end{tabular}

QUADRO 7

Coeficientes de correlação linear entre a porcentagem de óleo nas várias partes da semente

\begin{tabular}{|c|c|c|c|c|c|c|}
\hline \multirow{2}{*}{$\begin{array}{l}\text { Tamanho } \\
\text { da } \\
\text { semente }\end{array}$} & \multicolumn{2}{|c|}{$\begin{array}{c}\text { Correlação entre } \% \text { de } \\
\text { óleo na semente e na } \\
\text { amêndoa }\end{array}$} & \multicolumn{2}{|c|}{$\begin{array}{c}\text { Correlação entre \% de } \\
\text { oleo na semente e na } \\
\text { casca }\end{array}$} & \multicolumn{2}{|c|}{$\begin{array}{c}\text { Correlação entre \% de } \\
\text { óleo na amêndoa e na } \\
\text { casca }\end{array}$} \\
\hline & nf & $\mathbf{r}$ & $\mathbf{r}$ & nf & nf & $\mathbf{r}$ \\
\hline $\begin{array}{l}\text { Pequena } \\
\text { Média } \\
\text { Grande }\end{array}$ & $\begin{array}{l}7 \\
9 \\
6 \\
\end{array}$ & $\begin{array}{l}0,68^{*} \\
0,71^{*} \\
0,56\end{array}$ & $\begin{array}{l}0,46 \\
0,24 \\
0,23\end{array}$ & $\begin{array}{l}7 \\
9 \\
6 \\
\end{array}$ & $\begin{array}{l}7 \\
9 \\
6 \\
\end{array}$ & $\begin{array}{l}0,45 \\
0,27 \\
0,24 \\
\end{array}$ \\
\hline Total & 26 & $0,69 * *$ & 0,24 & 26 & 26 & $0,38 *$ \\
\hline
\end{tabular}

